\title{
Direct metallization by a plasma coating process on thermal conductive polyamide
}

\author{
Alexandru Sover ${ }^{*}$ and Jonas Amend \\ Ansbach University of Applied Sciences, Faculty of Engineering Sciences, Residenzstr. 8, Ansbach, \\ Germany
}

\begin{abstract}
This paper describes the metallization on highly filled thermal conductive Polyamide 6 material and the influence of the process conditions of the samples on the quality of the metal deposition. In a series of tests it is establish that the quality of the injection moulding process and fillers used for the polyamide material has a significant impact on the metal deposition on the polymeric surface. Even if the same surface roughness is generated by laser structuring the adhesion of the metallic particles decreases considerably as the quality of the polymeric surface is less perfect, i.e. when more fillers appear on the surface of the sample.
\end{abstract}

\section{Introduction}

Direct Metallization (DM) is an innovative plasma process to deposit metal on different substrates materials. This technology offers a great environmentally friendly alternative to the currently existing technologies of metal deposition on plastic materials and opens new options for different applications, in terms of design, surface protection, low-friction surfaces, and magnetic coatings [1-7]. The combination of plastic with metal on its surface can also improve the quality and functionality of components concerning thermal, electrical and electromagnetic properties, which is presented in different papers $[1-3,7,8]$.

The plastic parts processed by injection moulding with different $3 \mathrm{D}$ geometries can partially or completely be metalized using different metal powders with varying thicknesses $[2,3,6]$.

In the tests presented in this paper the Plasmadust ${ }^{\circledR}$ technique was used to apply metal layers on the surface of plastic parts. This is a thermal spray technology using cold active plasma to melt a metal powders such as copper, which can be deposited directly on a surface $[3,4]$. This can be described as additive metallization technology, which enables new applications also for non-metal materials like plastics.

In different papers $[2-4,6,8]$ the importance of the preparation of the deposition surface is described as a very important step for a high quality and adherence of the metallic layer. This plays a very important role, particularly on plastic materials which have a lower melting point than the metals and on which the higher deposition temperature can damage the surface. The manufacturing process of the plastic parts and the quality of the surface can influence the deposition quality of the plasma metallization. Some of the highly filled

\footnotetext{
*Corresponding author: a.sover@hs-ansbach.de
} 
plastic materials are very difficult to manufacture by injection moulding and offer only a poor surface condition for metallic depositions.

The aim of this work was to establish the influence of the surface quality after injection moulding process on the metallic deposition by thermal spray technology (Plasmadust ${ }^{\circledR}$ ) on the highly filled thermal conductive Polyamide 6.

\section{Materials, machine and preparation of samples}

The plastic material used on trials is a thermal conductive Polyamide 6 (ALCOM TCD PA6 5070 FR 15021) which provides significantly improved heat-conducting properties compared to standard plastics. The plastic material is thermally conductive and electrically insulating and offers an optimum heat management for efficient heat dissipation in different applications like lighting or circuit carriers. Its melting point is at about $220^{\circ} \mathrm{C}$ and the injection moulding melt temperature is between $280-320^{\circ} \mathrm{C}$. The polymer was dried at $80^{\circ} \mathrm{C}$ for two hours according to the technical data sheet. The residual moisture content was $0.0642 \%$ which - according to the recommendation - is enough for the processing by injection moulding.

The prepared samples have the dimensions of $150 \times 100 \mathrm{~mm}$ with a $4 \mathrm{~mm}$ thickness. The Polyamide 6 samples were prepared using the injection moulding machine Krauss Maffei, type KM CXL 130-750/380, with maximal clamping force $1300 \mathrm{kN}$. The mould is one cavity steel mould and it was tempered to $90^{\circ} \mathrm{C}$ during the injection moulding of the samples. Here a lower temperature was used than recommended for the mould temperature in the technical data sheet, which should be above $100^{\circ} \mathrm{C}$. The reason was to create a samples surface with different surface conditions. Some surface areas should have typical injection moulding failure as striations or different roughnesses. The samples are presented in the Figure 1, in which also the surface failure can be observed.

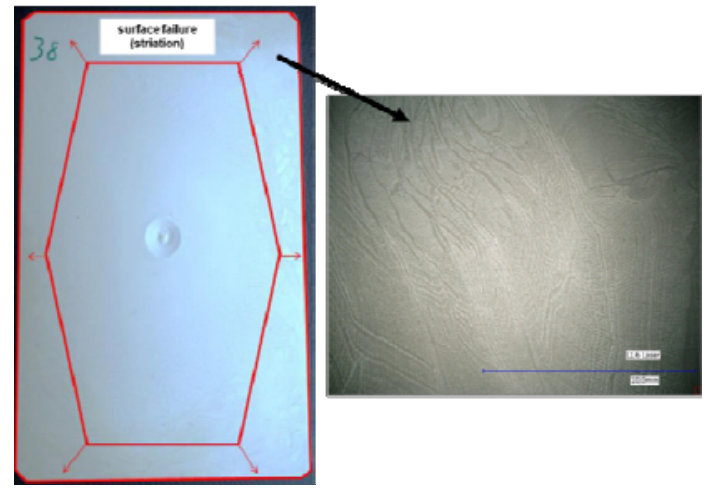

Fig. 1. Polyamide 6 injection moulding sample $(150 \times 100 \times 4 \mathrm{~mm})$ and surface failure (left).

The middle part of the sample close to the injection moulding gate, inside the red line, shows a very smooth, polished surface but the areas furthest from the injection point present different failures like striations. This surface failure type is typical for injection moulding process when the mould temperature is to low and the polymer starts to solidify during the filling of the cavity. This effect is more severe the more highly filled the polyamide material is. Under microscope the structure of the filler on the surface can be recognised. For a number of different plastic components these defects are irrelevant, but for visible components or further processing steps, like metallization, they can be an inconvenient.

The injected moulded plastic specimens should provide a certain surface to enable a good metallization. The laser structuring technology offers a flexible and accurate surface 
preparation, which substantially improves the metallization of the plastic materials and induces a better adhesion of the metallic layer $[1,5]$. The laser generates a certain structure on the plastic surface without thermal damage. In terms of roughness the structure can vary - depending on the laser parameters. The Figure 2 presents the polyamide sample with two defined lasered surfaces.

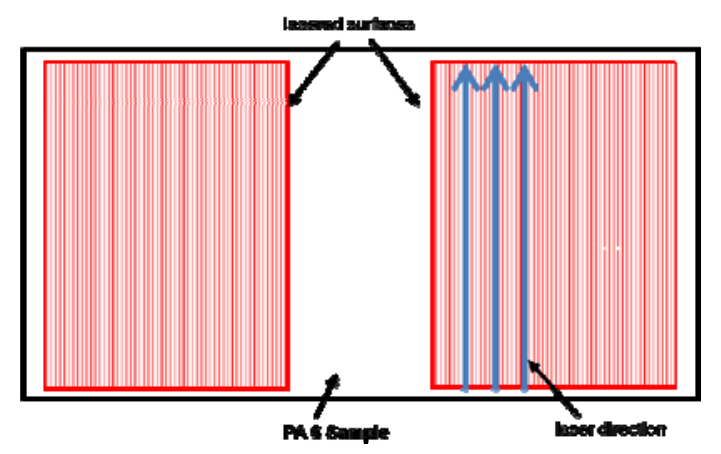

Fig. 2. Laser structuring on the surfaces of the PA samples.

For a better characterisation of the influence of the metallization process on injected moulded PA6, samples with and without failures on the same surface were used. Two big areas were structured (Figure 2) by laser using various parameters, presented in the Table 1.

Table 1. Laser parameters used for the structuring of the PA6 surface.

\begin{tabular}{|c|c|c|c|}
\hline Trials & Power $[\mathbf{W}]$ & Speed $[\mathbf{m m} / \mathbf{s}]$ & Structuring number \\
\hline L 1 & 5 & 300 & 1 \\
\hline L 2 & 15 & 300 & 1 \\
\hline L 3 & 5 & 1500 & 1 \\
\hline L 4 & 15 & 1500 & 1 \\
\hline L 5 & 15 & 300 & 2 \\
\hline L6 & 15 & 300 & 4 \\
\hline
\end{tabular}

The structuring of the surfaces was carried out at two different laser power levels and two different laser speeds, at an overlapping of 50\%. The sample in trial L5 was structured two times and in trial L6 four times to observe the influence of the repetition of structuring on the surface.

The metallization of the structured PA6 sample surfaces, was carried out by an atmospheric plasma machine, consisting of a plasma head which can move in different directions. The plasma head was moved in horizontal direction over the samples from top to bottom each time with a small offset, so that all the structured areas of the samples can be covered. The metallic powder used for the metallization is a special copper powder produced by ECKART GmbH (member of ALTANA Group) with the trade name Plasmavario 1001 Copper. This powder is a high purity copper (99.9\%) with very fine spherical particles (average particle size $10 \mu \mathrm{m}$ ) and narrow particle size distribution and a high electrical conductivity [6]. The copper powder is added to the plasma head where it melts under high temperature and from where it is projected with the argon gas onto the surface which should be metallised. To avoid thermal damage to the surface a certain distance between the plasma jet and surface is necessary, otherwise the polymeric surface can deteriorate and the adhesion of the "printed" structures is negatively affected. Two 
different distances were tested: $45 \mathrm{~mm}$ and $55 \mathrm{~mm}$. All other parameters for the plasma metallization were kept constant.

\section{Results}

The quality of the laser structuring on the plastic materials is an important parameter for the later metallization of the surface. The achieved roughness after laser structuring can provide a better adhesion for the first metallic layer on the polymer surface.

The Figure 3 shows different surface roughnesses of the PA6 polymer at different laser power levels and speeds.

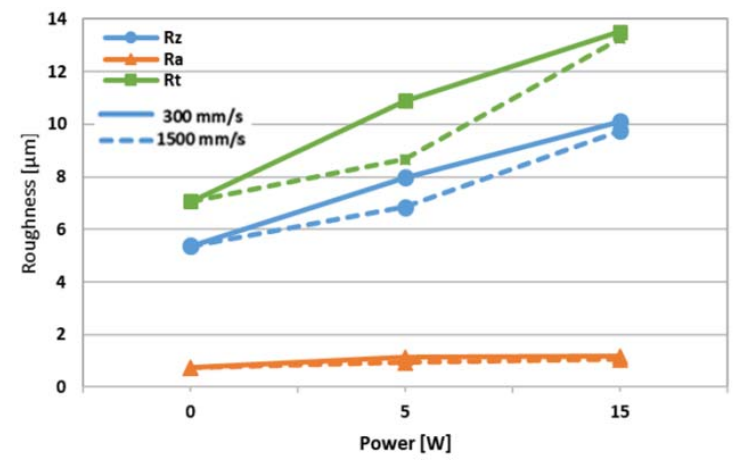

Fig. 3. Surface Roughness (Rz, Ra and Rt) before ( $0 \mathrm{~W})$ and after laser structuring at $300 \mathrm{~mm} / \mathrm{s}$ and $1,500 \mathrm{~mm} / \mathrm{s}$ laser speed and $5 \mathrm{~W}$ respectively $15 \mathrm{~W}$ power.

The profile roughness parameter $\mathrm{Rz}$ (average surface roughness), $\mathrm{Ra}$ (arithmetical mean deviations from the centre line) and Rt (maximum height of the profile) are commonly used to describe the roughnesses of a surface. The Figure 3 of the roughness measurements of the laser processed surfaces shows that the roughness of the surface of PA6 polymer increases with rising laser power regardless of the speed. Rz and Ra highlight this tendency and the maximum roughnesses are achieved at a power of $15 \mathrm{~W}$ and a speed of $300 \mathrm{~mm} / \mathrm{s}$, $\mathrm{Rz}=10.10 \mu \mathrm{m}$ respectively $\mathrm{Rt}=13.51 \mu \mathrm{m}$. The roughness of the processed surface is increased by approx. $100 \%$.

The Figure 4 shows the influence of the laser speed on the roughnesses Rz, Ra, Rt at $5 \mathrm{~W}$ laser power.

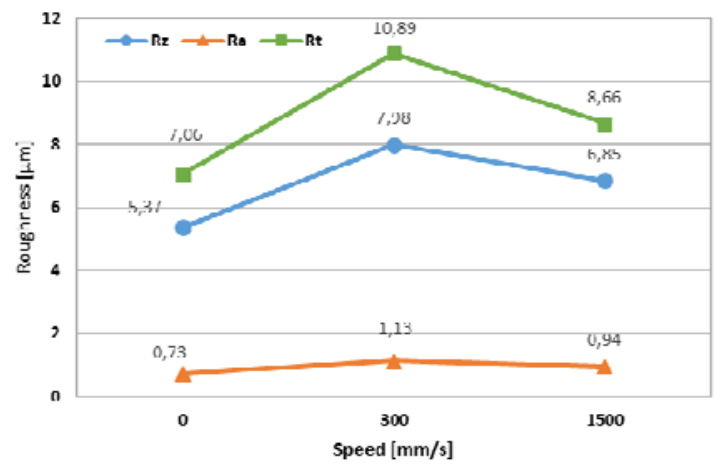

Fig. 4. Influence of the structuring speed on the surface roughness by $5 \mathrm{~W}$ laser power.

At $1500 \mathrm{~mm} / \mathrm{s}$ the roughness of the surface was lower than at $300 \mathrm{~mm} / \mathrm{s}$. The reason for this effect is that at higher speed the laser beam loses the energy which is necessary to 
structures the surface. The Figure 5 presents the roughness of the PA6 surfaces after several iterations of laser structuring.

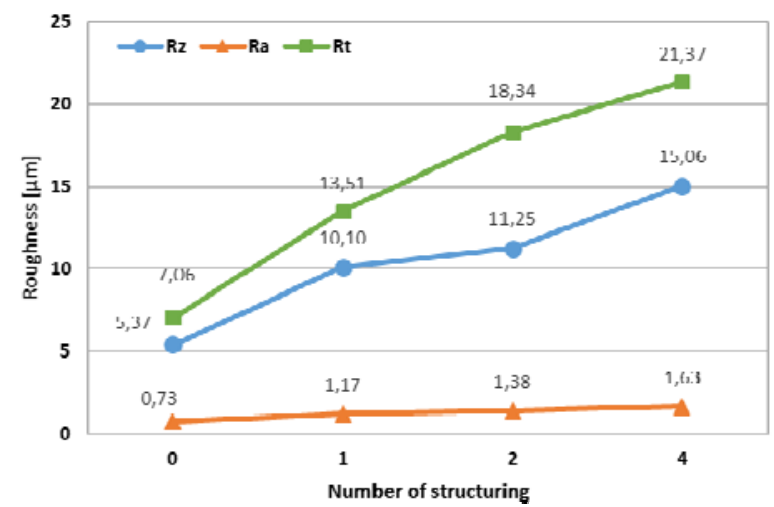

Fig. 5. Influence of the repetition of laser structuring on the surface roughness's $(v=300 \mathrm{~mm} / \mathrm{s}$, $\mathrm{P}=15 \mathrm{~W})$.

A higher number of repetitions increases the roughness of the surface. After four iterations the roughness of the surface increases by three times as compared to the roughness before the laser processing: $\mathrm{Rz} 5.37 \mu \mathrm{m}$ (before structuring) and $\mathrm{Rz} 15.06 \mu \mathrm{m}$ after four structuring steps. After all four laser structuring repetitions on the same surface of the samples no thermal damage is observed on the polymer surface, which can depreciate the metallic deposition on the surface.

The Figure 6 presents the PA6 sample after plasma metallization. The metal deposition is possible only on the laser structured areas of the sample. For the metallic deposition a certain roughness of the surface is necessary, where the first metallic layer/ particle can adhere. Where the surface is not structured by laser no metallic deposition is possible.

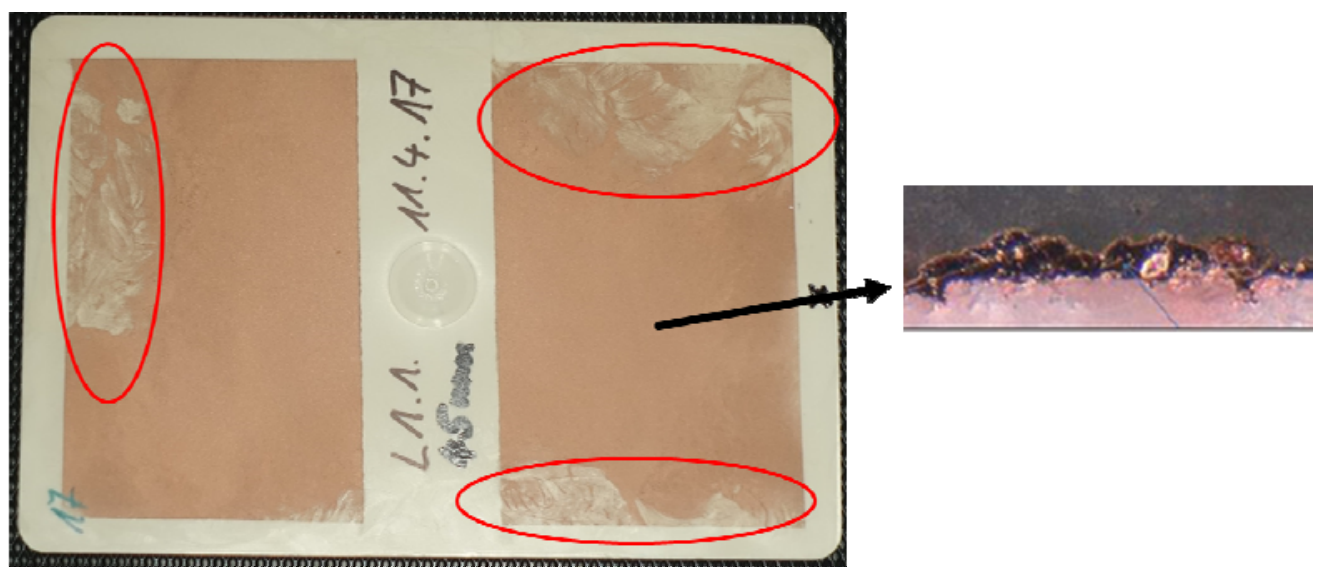

Fig. 6. Metallized PA plate with different surface failure $(45 \mathrm{~mm}$ distance between the plasma head and sample surface).

It is recognizable that the metallic deposition was not possible on areas with surface failure caused in the injection moulding process, presented in Figure 1. When comparing Figure 1 and 6 one can observe that the striations on the surface of the PA6 samples influence the metallic deposition by plasma drastically and even the structuring of the surface by laser cannot improve the deposition of the metallic particles. The melted/ softened cooper metallic powder cannot adhere on the PA6 surface if in processing failures 
on the surface occur, or the fillers are coming on the surfaces. The results of the cross cutting tests on the metallized samples show that the adhesion of metallic coating on the polymeric underground, for all roughnesses achieved by laser structuring and the used plasma head distances by metallization is good. No difference in the adhesion at a higher roughness was observed. The areas with surface failure are not to be tested because the metallic layer does not adhere and can be removed by the mere wiping of the surface. The distance between the plasma head and the samples is crucial for the thickness of the deposited layers: at $45 \mathrm{~mm}$ distance an increase between $12 \%$ und $30 \%$ in the thickness of deposition was observed. The thickness of the copper layer is higher (max. 16.2 $\mu \mathrm{m})$ at higher roughness $(\mathrm{Rz} 15.1 \mu \mathrm{m})$ of the laser structured surface.

\section{Conclusions}

The deposition of copper on PA6 polymeric substrates after different processing conditions (injection moulding and laser structuring) was tested. Without any surface structuring the adhesion of the copper substrate was not possible. After the laser structuring of the polymeric surfaces a metallic deposition was possible and the adhesion quality was not influenced by the surface roughnesses. The surface failures occurred in the injection moulding of the samples are decisive for the plasma metallization. On these areas a metal deposition was not possible even after the laser structuring. The different degrees of roughness achieved by laser structuring did not improve the adhesion of the copper layer on the examined PA polymers.

A special gratitude I give to the "kunststoffcampus bayern" in Weissenburg, which supported this project by providing the technical equipment. Special thanks for her support go to Ms Antje Sover for reviewing the article.

\section{References}

1. M. Mueller and J. Franke, $\mathbf{3 9}^{\text {th }}$ International Spring Seminar on Electronics Technology (ISSE), Pilsen, 43-47 (2016)

2. O.V. Penkov, M. Khadem, WS. Lim, et al. J Coat Technol Res 12, 225 (2015)

3. R. Gonzalez, H. Ashrafizadeh, A. Lopera, et al. J Therm Spray Tech 25, 897 (2016)

4. J. Franke, A. Syed-Khajaa, R. Schramm, R. Ochs, Procedia CIRP 37, 59-64 (2015)

5. R. Schramm and J. Franke, IEEE $\mathbf{1 5}^{\text {th }}$ Electronics Packaging Technology Conference (EPTC 2013), Singapore, 108-113 (2013)

6. A. Sover, P. Popp and T. Aydin, MATEC Web of Conferences 112 (2017)

7. P.L. Fauchais, J.V.R. Heberlein, M.I. Boulos, Industrial Applications of Thermal Spraying Technology, Thermal Spray Fundamentals. (Springer, Boston, MA, 2014)

8. A. Małachowska, M. Winnicki, Ł. Konat, T. Piwowarczyk, L. Pawłowski, A. Ambroziak, M. Stachowicz, Surface and Coatings Technology, 318, 82-89 (2017) 